\title{
Lipofilling Outcomes Mimicking Breast Cancer Recurrence: Case Report and Update of the Literature
}

\author{
SILVIA GIGLI ${ }^{1}$, MARIA IDA AMABILE ${ }^{2}$, FRANCESCA DI PASTENA ${ }^{1}$, ALESSANDRO DE LUCA ${ }^{2}$, \\ CATERINA GULIA $^{3}$, LUCIA MANGANARO ${ }^{1}$, MASSIMO MONTI $^{2}$ and LAURA BALLESIO ${ }^{1}$ \\ Departments of ${ }^{1}$ Radiology, Anatomo-pathology and Oncology, ${ }^{2}$ Surgical Sciences, and \\ ${ }^{3}$ Gynecology, Obstetrics and Urology, Sapienza University of Rome, Rome, Italy
}

\begin{abstract}
Breast lipofilling uses autologous fat grafting to correct breast defects after radical or conservative surgery. After early concerns regarding its application in reconstruction after breast cancer (BC), in 2009 the American Society of Plastic Surgeons formed a task force to assess the indications, safety and efficacy of autologous fat grafting. We report the case of a woman who came to our attention for a painful swelling of the left breast. She had undergone breast-conserving therapy for BC, followed by lipofilling. The breast ultrasound (US) examination showed diffuse structural alteration and multiple hypoechoic areas with acoustic shadowing, mainly localized in the subcutaneous tissue. After pharmacological treatment and shortterm follow-up US examination, considering the persistence of the clinical symptoms and structural alterations, we performed contrast-enhanced magnetic resonance imaging, that showed multiple enhancing areas in the left breast. Suspecting local tumor recurrence, we carried out US-guided breast core-biopsy, whose histological examination documented liponecrosis. This observation raised a series of diagnostic and therapeutic issues highlighting the diagnostic pitfalls that the radiologist may encounter during the evaluation of patients who have undergone $B C$ surgery and breast reconstruction through lipofilling.
\end{abstract}

Lipofilling is a reconstructive and esthetic technique that is being increasingly used in breast surgery, and uses autologous fat tissue, taken to increase breast volume from other locations and to improve breast consistency and profile (1). The first description of the use of autologous fat graft as

Correspondence to: Maria Ida Amabile, MD, Ph.D., Department of Surgical Sciences, Sapienza University of Rome, Viale Regina Elena 324, 00161 Rome, Italy. E-mail: marida.amabile@gmail.com

Key Words: Breast cancer, lipofilling, inflammation, breastconserving therapy, ultrasound exam, contrast-enhanced magnetic resonance imaging. a filler for correcting defects and remodeling contours was reported in 1895 by Czerny (2), who augmented the breast volume of a patient with a lipoma removed from her own lumbar region. In 1987, Bircoll was the first to describe a procedure which combined liposuction and autologous fat tissue transplantation in the breast (3), with the major advantage of virtually limitless donor tissue. At present, the most frequently used technique for lipofilling is that described by Coleman in 1995 (4), in which the harvested fat tissue is stored in syringes, centrifuged in a closed system, obtaining concentrated viable fat cells, after discarding the supernatant and the lower layers, which is essential for successful fat grafting. Initially, concerns regarding the development of complications such as fat necrosis, oil cyst formation and calcifications, that could compromise the early detection of breast cancer, led to wide skepticism in the application of the lipofilling method $(5,6)$. In 2009, a task force of the American Society of Plastic Surgeons (ASPS) assessed the efficacy and safety of lipofilling in 283 patients, most of them showing satisfactory results in a follow-up time from 1 month to 10 years (7). The ASPS made recommendations for the safe and efficacious use of fat grafting to the breast (7).

Our recent observation of a patient who had undergone breast-conservative therapy for breast cancer followed by lipofilling, and presented with suspicious findings of local tumor recurrence, raised a series of diagnostic and therapeutic issues persuading us to carry out a review of the literature focusing on the diagnostic imaging pitfalls that may occur during cancer follow-up after breast lipofilling.

\section{Case Report}

A 57-year-old Caucasian woman came to our attention referring a painful swelling in the outer quadrants of the left breast. More than 2 years earlier, the patient had been treated for invasive ductal breast carcinoma, for which she underwent 
lumpectomy of the left breast followed by radiation therapy. Subsequently, the patient had undergone breast reconstruction with lipofilling. The breast ultrasound (US) examination showed a diffuse structural modification and the presence of multiple hypoechoic areas with acoustic shadowing, mainly localized in the subcutaneous adipose tissue (Figure 1). Because of the doubtful interpretation of the image, a shortterm follow-up US examination was performed 3 months later, highlighting no improvement in the structural framework and indicating the need for further investigation with breast contrast-enhanced (CE) magnetic resonance imaging (MRI). The T1 and T2 MRI sequences confirmed a diffuse structural modification of the left breast, together with severe edema of the breast tissue. The post-contrast images showed multiple enhancing areas, some of them confluent, with blurred margins and maximum diameter of about $1 \mathrm{~cm}$ (Figure 2). A short anti-inflammatory oral treatment to reduce breast pain and tissue edema was prescribed and, after it, a second-look US examination was performed. Because the lesions still persisted and were clearly visible, indicating a strong suspicious of malignancy, an US-guided breast core-needle biopsy was performed (at two different sites) (Figure 3). The histological examination documented an inflammatory infiltrate and fat necrosis, in the absence of any cellular atypia.

\section{Discussion}

Patient expectations for a natural breast shape after breast surgery, whether conservative or radical, are high (8-12). Lipofilling has been recently described as a low-risk procedure with low-morbidity which gives good results for the correction of soft-tissue defects $(13,14)$. After early concerns that this procedure would cause scarring in the breast and interfere with breast screening examination $(5,6)$, the ASPS Fat Graft Task Force was formed in 2009 to assess the safety and efficacy of the procedure (7). The work performed by the ASPS Fat Graft Task Force documented that, during the literature search regarding breast lipofilling, mostly small case series, case reports, and expert opinions were available, describing fat grafting for various breast indications, both cosmetic and reconstructive, and, combined together, for a total of 283 patients having fat grafting procedures, with a follow-up from 1 month to 10 years (7). The risk of malignancy with lipofilling could not be identified due to lack of larger randomized controlled trials and lack of standardized techniques. Total complications comprised of $12.7 \%$ (7). The most frequent complication was liponecrosis. Among different studies, the incidence of fat necrosis was, however, extremely variable, also depending on the lipofilling technique used (15-17). Usually liponecrosis is asymptomatic, sometimes patients may present local ecchymosis or a palpable mass. On mammographic examination, liponecrosis may appear as a radiolucent rounded image surrounded by a

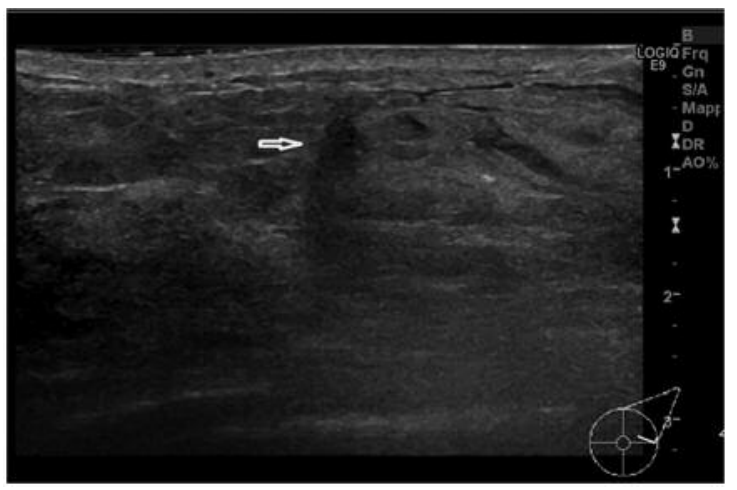

Figure 1. Ultrasound imaging showing diffuse edema and hypoechoic area with acoustic shadowing in the outer quadrants of the left breast.

thin radiopaque rib $(16,17)$. The presence of calcifications on mammographic examination may lead to a suspicion of breast cancer relapse, especially at the first stage of the process when the calcifications present small size $(17,18)$. The imaging findings on US examination may be misleading, presenting as a structural inhomogeneity of the subcutaneous fat tissue or a hypoechoic aspect with blurred margins and posterior acoustic shadowing (16). In our experience, the breast US finding of structural modification and the presence of multiple hypoechoic areas with blurred margins and acoustic shadowing, together with the MRI finding of multiple enhancing nodules, led us to a high suspicion for cancer relapse (19). Perhaps mammographic examination could have been helpful in discriminating between liponecrosis and disease relapse, but we were not able to perform this due to the intense pain in the patient's left breast which did not allow adequate compression.

At MRI, fat necrosis presents different findings depending on the stage of the process (20). Usually, it appears as a mass with round or oval shape, isointense relative to fat, showing high signal on T1-weighted non-fat saturated images, hyperintense on T2-weighted non-fat saturated images, and hypointense on T2-weighted fat-saturated images (20). The degree of lesion enhancement, which usually appears as a rim enhancement, depends on the stage (acute or chronic) of the inflammatory reaction. Recent lesions present irregular margins and may have variable enhancement surrounding the lesion, while older lesions show marked irregularity, retraction, fibrosis and generally do not enhance after administration of contrast medium $(16,20)$.

Our patient presented multiple enhancing nodules in the left breast on the post-contrast images. When an enhancement is identified in a breast gland treated for cancer, a core-needle biopsy appears to be essential in order to exclude recurrence. The adipose tissue produces various pro- and antiinflammatory adipokines to modulate inflammation and insulin 


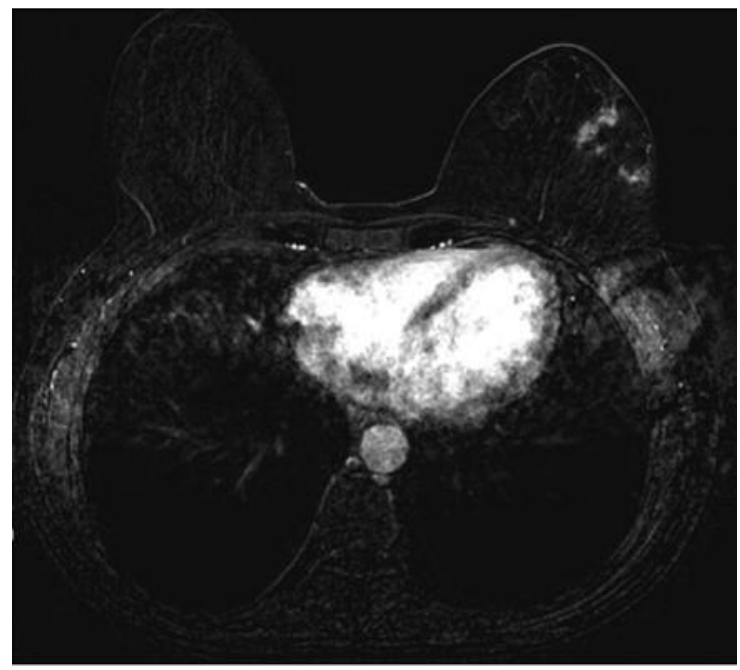

Figure 2. Contrast-enhancement magnetic resonance imaging showing multiple enhancing nodules, some of them confluent, with blurred margins and maximum diameter of about $1 \mathrm{~cm}$ in the left breast.

resistance $(21,22)$. Leptin, and other adipokines, are able to activate monocytes and macrophages to produce inflammatory interleukin (IL)-6, tumor necrosis factor- $\alpha$ and IL12 (23). Moreover, experimental studies have shown that several adipokines can stimulate breast cancer cells through endocrine, paracrine and autocrine pathways (22). In theory, the interaction between the tumor and the stroma tissue may potentially induce cancer recurrence by fueling dormant cancer cells in the tumor bed (14). Manabe et al. demonstrated that adipocytes increase proliferation of breast cancer cells in vitro (24). Iyengar et al. found that adipocytes increased cell proliferation and the invasive potential of malignant breast epithelial cells in vitro (25). Zocchi and Zuliani followed-up 181 patients who had undergone breast lipofilling after breast surgery for cancer, and did not report any cases of de novo carcinogenesis in a follow-up period of 10 years after the lipofilling procedure (26).

We performed an US-guided core-needle biopsy of the left breast, which revealed the presence of a severe inflammatory reaction and fat necrosis, while no cellular atypia was found. The inflammation was confined to the harvested fat injection sites, which did not resolve after conservative medical therapy. The local inflammation that may occur in a breast treated with lipofilling after breast cancer surgery can cause significant diagnostic difficulties in the follow-up investigations. Certainly, the degree of inflammation depends on the lipofilling technique used and on patient's intrinsic characteristics, such as immune reactivity. However, inflammatory tissue has some imaging characteristics that are similar to those of cancer.

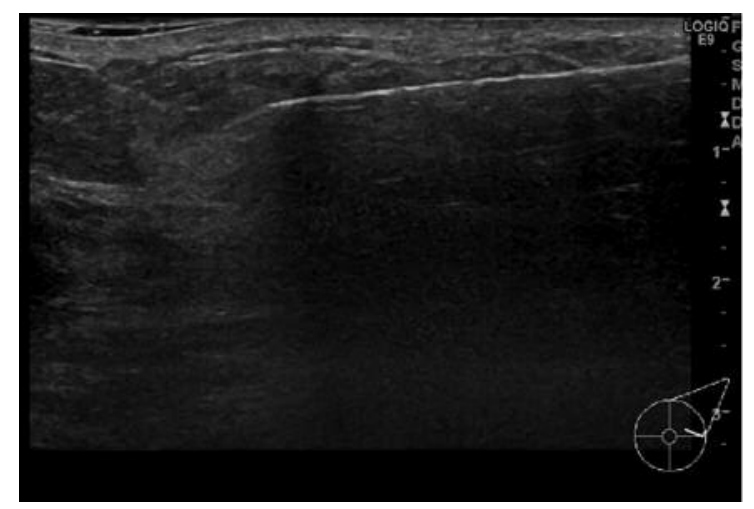

Figure 3. Ultrasound-guided breast core-needle biopsy of the structural alterations persisting in the outer quadrants of the left breast after oral anti-inflammatory therapy.

In conclusion, the experience of the past two decades has confirmed lipofilling to be a safe reconstructive technique. However, it would be appropriate to consider the tissue modifications that occur after this procedure which can lead to diagnostic pitfalls and difficulties in the clinical management and diagnostic follow-up of patients with previous breast cancer.

\section{Disclosure}

The Authors have stated that they have no conflicts of interest in regard to this study.

\section{References}

1 Wazir U, El Hage Chehade H, Headon H, Oteifa M, Kasem A and Mokbel K: Oncological safety of lipofilling in patients with breast cancer: a meta-analysis and update on clinical practice. Anticancer Res 36: 4521-4528, 2016.

2 Czerny V: Plastischer ersatz der brustdruse durch ein lipom. Verhandl Deutsche Ges Chir 24: 216-217, 1895.

3 Bircoll M: Cosmetic breast augmentation utilizing autologous fat and liposuction techniques. Plast Reconstr Surg 79: 267-271, 1987.

4 Coleman SR: Long-term survival of fat transplants: controlled demonstrations. Aesthetic Plast Surg 19: 421-425, 1995.

5 Kneeshaw PJ, Lowry M, Manton D, Hubbard A, Drew PJ and Turnbull LW: Differentiation of benign from malignant breast disease associated with screening detected microcalcifications using dynamic contrast enhanced magnetic resonance imaging. Breast 15: 29-38, 2006.

6 Pierrefeu-Lagrange AC, Delay E, Guerin N, Chekaroua K and Delaporte T: Radiological evaluation of breasts reconstructed with lipomodeling. Ann Chir Plast Esthet 51: 18-28, 2006.

7 Gutowski KA and ASPS FAT GRAFT TASK FORCE: Current applications and safety of autologous fat grafts: A report of the ASPS Fat Graft Task Force. Plast Reconstr Surg 124: 272-280, 2009. 
8 Amabile MI, Monti M, Merola R, Frusone F, D'Orazi V and Pasta V: Nipple-sparing mastectomy in patients with preoperative diagnosis of non-invasive breast carcinoma. A single-center experience. Anticancer Res 36: 779-783, 2016.

9 Amabile MI, Mazouni C, Guimond C, Sarfati B, Leymarie N, Cloutier AS, Bentivegna E, Garbay JR, Kolb F and Rimareix F: Factors predictive of re-excision after oncoplastic breastconserving surgery. Anticancer Res 35: 4229-4234, 2015.

10 Pasta V, D’Orazi V, Merola R, Frusone F, Amabile MI, De Luca $\mathrm{A}$, Buè R and Monti M: Oncoplastic central quadrantectomies. Gland Surg 5: 422-426, 2016.

11 Qureshi AA, Odom EB, Parikh RP, Myckatyn TM and Tenenbaum MM: Patient-reported outcomes of aesthetics and satisfaction in immediate breast reconstruction after nipplesparing mastectomy with implants and fat grafting. Aesthet Surg J, 2017. doi: 10.1093/asj/sjx048. [Epub ahead of print]

12 Tardioli S, Ballesio L, Gigli S, DI Pastena F, D’Orazi V, Giraldi G, Monti M, Amabile MI and Pasta V: Wire-guided localization in non-palpable breast cancer: results from monocentric experience. Anticancer Res 36: 2423-2427, 2016.

13 Cigna E, Ribuffo D, Sorvillo V, Atzeni M, Piperno A, Calò PG, and Scuderi N: Secondary lipofilling after breast reconstruction with implants. Eur Rev Med Pharmacol Sci 16: 1729-1734, 2012.

14 Hamza A, Lohsiriwat V and Rietjens M: Lipofilling in breast cancer surgery. Gland Surg 2: 7-14, 2013.

15 Chirappapha P, Rietjens M, De Lorenzi F, Andrea M, Hamza A, Petit JY, Garusi C, Martella S, Barbieri B and Gottardi A : Evaluation of lipofilling safety in elderly patients with breast cancer. Plast Reconstr Surg Glob Open 3: e441, 2015.

16 Atasoy MM, Oren NC, Ilica AT, Güvenç İ, Günal A and MossaBasha M: Sonography of fat necrosis of the breast: correlation with mammography and MR imaging. J Clin Ultrasound 41: 415-423, 2013.

17 Gigli S, Amabile MI, Di Pastena F, Manganaro M, David E, D’Orazi V, Monti M, Catalano C and Ballesio L: Magnetic resonance imaging after breast oncoplastic surgery: an update. Breast Care, 2017. doi: 10.1159/000477896. [Epub ahead of print]

18 Chala LF, de Barros N, de Camargo Moraes P, Endo E, Kim SJ, Pincerato KM, Carvalho FM and Cerri GG : Fat necrosis of the breast: mammographic, sonographic, computed tomography, and magnetic resonance imaging findings. Curr Probl Diagn Radiol 33: 106-126, 2004
19 Ballesio L, Savelli S, Angeletti M, Porfiri LM, D’Ambrosio I, Maggi C, Castro ED, Bennati P, Fanelli GP, Vestri AR and Manganaro L: Breast MRI: Are T2 IR sequences useful in the evaluation of breast lesions? Eur J Radiol 71: 96-101, 2009.

20 Kerridge WD, Kryvenko ON, Thompson A and Shah BA: Fat necrosis of the breast: a pictorial review of the mammographic, ultrasound, CT, and MRI findings with histopathologic correlation. Radiol Res Pract 2015: 613139, 2015.

21 Kwon H and Pessin JE: Adipokines mediate inflammation and insulin resistance. Front Endocrinol 4: 71, 2013.

22 Molfino A, Amabile MI, Monti M, Arcieri S, Rossi Fanelli F and Muscaritoli M: The role of docosahexaenoic acid (DHA) in the control of obesity and metabolic derangements in breast cancer. Int J Mol Sci 17: 505, 2016.

23 Gainsford T, Willson TA, Metcalf D, Handman E, McFarlane C, $\mathrm{Ng}$ A, Nicola NA, Alexander WS and Hilton DJ: Leptin can induce proliferation, differentiation, and functional activation of hemopoietic cells. Proc Natl Acad Sci USA 93: 14564-14568, 1996.

24 Manabe Y, Toda S, Miyazaki K and Sugihara H: Mature adipocytes, but not pre-adipocytes, promote the growth of breast carcinoma cells in collagen gel matrix culture through cancerstromal cell interactions. J Pathol 201: 221-228, 2003.

25 Iyengar P, Combs TP, Shah SJ, Gouon-Evans V, Pollard JW, Albanese C, Flanagan L, Tenniswood MP, Guha C, Lisanti MP, Pestell RG and Scherer PE: Adipocyte-secreted factors synergistically promote mammary tumorigenesis through induction of anti-apoptotic transcriptional programs and protooncogene stabilization. Oncogene 22: 6408-6423, 2003.

26 Zocchi ML and Zuliani F: Bicompartmental breast lipostructuring. Aesthetic Plast Surg 32: 313-328, 2008.
Received July 28, 2017

Revised August 12, 2017

Accepted August 21, 2017 